\title{
Study of the Coordination of Quinuclidine to a Chiral Zinc Phthalocyanine Dimer
}

\author{
Nelson Giménez-Agullóa, Gemma Aragay ${ }^{a}$, José Ramón Galán-Mascarós ${ }^{\star a, b}$ and \\ Pablo Ballester ${ }^{*}, \mathrm{~b}$
}

\author{
We dedicate this work to Prof. Tomás Torres, Universidad Autónoma de Madrid on the occasion of his $65^{\text {th }}$ \\ anniversary. \\ ${ }^{a}$ Institute of Chemical Research of Catalonia (ICIQ), The Barcelona Institute of Science and Technology, Av. Països \\ Catalans 16, 43007 Tarragona,Spain; e-mail: pballester@iciq.es ${ }^{b}{ }^{2}$ stitution for Research and Advanced Studies \\ (ICREA), Passeig Lluis Companys, 23, 08010 Barcelona, Spain
}

Received date (to be automatically inserted after your manuscript is submitted) Accepted date (to be automatically inserted after your manuscript is accepted)

\begin{abstract}
We attempted the calculation of an accurate equilibrium constant for the dimerization process of enantiomerically pure Zn-1 using UV-vis dilution experiments. At millimolar concentration Zn-1 is involved in a chemical exchange process between its monomeric and dimeric state that is slow on the ${ }^{1} \mathrm{H}$ NMR timescale. We performed variable-temperature ${ }^{1} \mathrm{H}$ NMR experiments in $\mathrm{CDCl}_{3}$ solution to determine the dimerization constant value at different temperatures and performed a van't Hoff plot to derive the thermodynamic parameters of the process. The calculated thermodynamic data revealed that the dimerization process is entropy-driven and enthalpically opposed. We also probed the coordination of quinuclidine, 1-azabicyclo[2.2.2] octane, 2, to the Zn-phthalocyanine using UV-vis and ${ }^{1} \mathrm{H}$ NMR titrations in $\mathrm{CDCl}_{3}$ solution. At micromolar concentration the $\mathbf{Z n - 1}$ exclusively exists in solution as a monomer and forms a simple $1: 1, \mathbf{2} \bullet \mathbf{1 - Z n}$, complex with quinuclidine having a stability constant of $K(\mathbf{2} \bullet \mathbf{1}-\mathbf{Z n})=10^{6} \mathrm{M}^{-}$ ${ }^{1}$. On the other hand, the ${ }^{1} \mathrm{H}$ NMR titrations carried out at $298 \mathrm{~K}$ and at millimolar concentration showed that Zn-1 was present in solution as the dimer and formed $2: 1, \mathbf{2} \bullet(\mathbf{1 - Z n})_{2}$, and $2: 2, \mathbf{2}_{2} \bullet(\mathbf{1}-\mathbf{Z n})_{2}$, complexes by coordination to $\mathbf{2}$. In addition, the $1: 1$ complex, $\mathbf{2} \bullet(\mathbf{1 - Z n})$, showed a reduced dimerization constant compared to the uncoordinated parent monomer 1-Zn. At high quinuclidine concentration, the 1:1 complex, 2•1-Zn, derived from the coordinated dimer dissociation was also detected. The ${ }^{1} \mathrm{H}$ NMR spectra of the titrations displayed separate signals for some hydrogen atoms of the Zn-phthalocyanine in each one of the four species. Remarkably, the chemical exchange processes involving free and bound quinuclidine in the monomeric and dimeric complexes showed different kinetics on the NMR timescale.
\end{abstract}

KEYWORDS: phthalocyanines, dimerization, coordination, quinuclidine, zinc

\footnotetext{
*Correspondence to: Prof. P. Ballester, Institution for Research and Advanced Studies (ICREA) and Institute of Chemical Research of Catalonia (ICIQ), The Barcelona Institute of Science and Technology, Av. Països Catalans 16, 43007, Tarragona (Spain), Fax (+34)977-920-211, e-mail: pballester@iciq.es
} 


\section{INTRODUCTION}

Phthalocyanines $(\mathrm{Pc})$ and their metallated derivatives have attracted general attention due to their interesting physical, electronic and optical properties. Of particular interest to this work is their tendency to aggregate by stablishing intermolecular $\pi-\pi$ stacking interactions. This ability provides Pcs with the capacity to self-assemble into complex supramolecular systems featuring new properties that are not present in the individual entities. The molecular aggregation of Pcs usually leads to one-dimensional nanostructures such as, nanoribons [1] or nanofibers [2] with interest in the field of functional materials. In recent years, the exceptional properties featured by the Pcs and their aggregates have found application in multiple fields that cover from gas sensors [3], molecular magnets [4], photovoltaic cells [5], photosensitizers for photodynamic therapy [6, 7], to non-linear optical materials [8].

Up to date, a large number of different substituted Pcs have been synthesized and their aggregation properties reported [9]. However, the number of reports dealing with chiral Pcs is scarce [10,11]. The unique optical and magnetic properties of chiral porphyrinoids make them interesting candidates for several applications including chiral recognition [12], chiral memory [13], and asymmetric catalysis [14]. Different approaches have been used for their synthesis leading to three diverse groups of optically active Pcs: 1) Pcs substituted with alkyl chains that contain a stereogenic carbon [15], 2) Pcs substituted with optically active components [16] and 3) Pcs displaying supramolecular asymmetry [17]. The most widely synthetic approach used consists on the introduction of chiral residues into the macrocycle's periphery. Chiral phthalocyanine Zn-1 (Figure 1) was reported in 2012 by Jiang and coworkers [18]. It constitutes a nice example of building an enantiomerically pure Pc through peripheral decoration with chiral binaphtyl units. It was described that $\mathbf{Z n}-\mathbf{1}$ formed dimeric aggregates held together through multiple cooperative $\pi-\pi$ interactions. The existence of $\mathbf{Z n - 1}$ as discrete dimeric entities (i.e. $[\mathbf{Z n - 1}]_{2}$ ) featuring a face-to-face supramolecular structure was demonstrated in the solid state by X-ray diffraction of single crystals and in solution using ${ }^{1} \mathrm{H}$ NMR spectroscopy techniques.

Metal coordination bonds have been extensively used to direct the self-assembly of large supramolecular architectures. In this context, transition-metal porphyrins have been amply exploited as acceptor building blocks since the central metal atom possesses at least one axial site available for coordination $[19,20]$. In turn, the use of metallophthalocyanines as acceptor ligands is very attractive not only due to their analogous structure with porphyrin complexes but also due to their higher chemical and thermal stability. Some works regarding the coordination of amino ligands to several $\mathrm{Zn}$ (II) phthalocyanines have been published in literature [21, 22, 23]. However, in these reports the determination of accurate binding constants for the corresponding complexes was not addressed in detail.

Herein we report the thermodynamic characterization of the aggregation process experienced by the chiral $\mathbf{Z n - 1}$ in $\mathrm{CHCl}_{3}$ solution. We show that the aggregation process of racemic $\mathbf{Z n - 1}$ does not lead to exclusive formation of a dimer. We also describe detailed studies of the coordination processes of enantiopure and racemic Zn-1 with quinuclidine, a nitrogenated ligand known to axially coordinate $\mathrm{Zn}$-metallated porphyrinoids. These studies were performed using two very different monomer concentrations, $\mu \mathrm{M}$ and $\mathrm{mM}$, in order to dissect the coordination processes of the monomeric and dimeric states of $\mathbf{Z n - 1}$. When coordinated to quinuclidine the thermodynamic stability of the $\mathbf{2}_{2} \bullet[\mathbf{Z n}-\mathbf{1}]_{2}$ dimer is reduced compared to that of the non-coordinated counterpart. The $\mathbf{2}_{2} \bullet(R, R)$ - or $(S, S)-[\mathbf{Z n}-\mathbf{1}]_{2}$ homodimers and the meso $(R, S)$-heterodimer are isoenergetic.

\section{Insert Figure 1}




\section{RESULTS AND DISCUSSION}

Synthesis of Zn-1: Chiral phthalocyanines $(R)$ - and $(S)$-Zn-1 (Figure 1) and the enantiomerically pure $(R)$ - and (S)dinitriles precursors were synthesized following reported procedures [18, 24]. The circular dichroism (CD) spectra of the prepared dinitriles, not reported in the original paper, showed, as expected, bisignated CD spectra due to the exciton coupling between the chromophores (naphthyls) present in the molecule.

The enantiopure dinitriles were refluxed in separated hexanol solutions in the presence of $\mathrm{Zn}(\mathrm{OAc})_{2}$ and DBU to yield the $(R)-\mathbf{Z n}-\mathbf{1}$ and $(S)-\mathbf{Z n - 1}$ [25]. Enantiopure Zn-1 was isolated in acceptable yields (39-45 \%) after silica column chromatography purification of the reaction crude using $\mathrm{CH}_{2} \mathrm{Cl}_{2}$ :THF (100:2) as eluent mixture. As expected, both enantiomers presented identical ${ }^{1} \mathrm{H}$ NMR and UV-vis spectra that were in agreement with the ones previously reported by Jiang and co-workers [18].

\section{Thermodynamic characterization of the dimerization process of $\mathrm{Zn}-\mathbf{1}$ :}

The ${ }^{1} \mathrm{H}$ NMR spectrum of $(R)-\mathbf{Z n}-\mathbf{1}$ displayed two singlets resonating at 9.30 and $7.83 \mathrm{ppm}$ assigned to the aromatic protons $\left(\mathrm{H}_{\mathrm{a}}\right.$, see Figure 1 for proton assignment) of the isoindole units and two sets of six signals appearing in the range of 8.72 to $6.79 \mathrm{ppm}$ corresponding to the aromatic protons of the peripheral binaphtyl moieties. This observation was in agreement with the described $\pi-\pi$ dimerization experienced by $(R)-\mathbf{Z n}-\mathbf{1}$ at $1 \mathrm{mM}$ concentration, that assigns a $C_{4}$ symmetry of the monomer in the dimeric assembly $[(R)-Z n-1]_{2}[26]$. The ${ }^{1} \mathrm{H}$ NMR spectrum recorded for a more diluted sample of $(R)-\mathbf{Z n}-\mathbf{1}$ in $\mathrm{CDCl}_{3}$ solution $\left(5 \times 10^{-4} \mathrm{M}\right)$ resulted in the emergence of a new set of proton signals that was attributed to the monomeric species, $(R)-\mathbf{Z n - 1}$, featuring $D_{4}$ symmetry. In the $(R)-\mathbf{Z n - 1}$ monomer the protons in the isoindole units appear as a singlet at $9.43 \mathrm{ppm}$. Moreover, the protons corresponding to the peripheral binaphthyl substituents produce a single set of six signals between 8.17-7.36 ppm owing to the symmetry increase for the monomer. A dimerization constant value of $K_{D}=7.3 \times 10^{3} \mathrm{M}^{-1}$ in $\mathrm{CDCl}_{3}$ was previously reported by Jiang et al. This constant value was calculated using the integral areas of the proton signals assigned to the monomer and the dimer.

Considering that the accurate assessment of the dimerization constant for enantiopure Zn-1 was crucial for the calculation of its speciation (monomer/dimer) in solution at any concentration, we decided to investigate the dimerization equilibria of $\mathbf{Z n - 1}$ in chloroform using dilution UV-vis experiments.

The aggregation of zinc-phthalocyanines is known to occur in solution even at the low concentrations used to register their UV-vis spectra [18]. The UV-vis spectrum of enantiopure $\mathbf{Z n - 1}$ in $\mathrm{CHCl}_{3}$ solution at a $10^{-6} \mathrm{M}$ concentration displayed the typical absorption bands of a metallated phthalocyanine (i.e. Soret band with maximum at $350 \mathrm{~nm}$, and an intense Q-band centered at $680 \mathrm{~nm}$ together with two weak shoulders with maxima at 616 and $647 \mathrm{~nm}$ ). The absorption band corresponding to the binaphtyl moieties was centered at $280 \mathrm{~nm}$. UV-vis dilution studies of enantiopure Zn-1 dissolved in chloroform in a concentration range of $10^{-5}$ to $10^{-6} \mathrm{M}$ returned absorption spectra that did not show significant changes in the epsilon scale. This result evidenced that in the used range of concentrations enantiopure $\mathbf{Z n - 1}$ existed in solution as a unique species, most likely the monomer considering the previously reported $K_{\mathrm{D}}$ value. Conversely, the UV-vis spectra acquired in a range of more concentrated solutions of enantiopure Zn-1 $\left(1 \times 10^{-5} \mathrm{M}\right.$ to $1.24 \times 10^{-4} \mathrm{M}$ ) showed a non-linear relationship between concentration and molar extinction coefficient values. Upon increasing the concentration of enantiopure Zn-1, the UV-vis spectrum (epsilon scale) displayed a small increment in the intensity of the bands centered at 617 and $647 \mathrm{~nm}$, while the band at $680 \mathrm{~nm}$ decreased in intensity (Figure 2). This observation suggested the formation of aggregates in solution induced by $\pi-\pi$ stacking interactions [18]. We performed the mathematical analysis of the UV-vis dilution data using a simple theoretical dimerization model. The fit of the experimental data to the model was good and returned a value of $K_{D}=5.17 \times 10^{3} \mathrm{M}^{-1}$ for the dimerization constant (Figure S1). 
It is worthy to note that the magnitude of the calculated $K_{D}$ should allow the observation of monomeric and dimeric species in the ${ }^{1} \mathrm{H}$ NMR spectra at the working concentration range $(2 \mathrm{mM})$. The observation of a single species (dimer) in the ${ }^{1} \mathrm{H}$ NMR spectra at this concentration indicated that the dimerization constant value was underestimated. Probably, the low amount of dimer present in solution in the range of concentrations covered for the UV-vis dilution experiments (i.e. less than 20\%) complicated the determination of an accurate value. However, the high absorptivity displayed by enantiopure Zn-1 did not allow us to acquire UV-vis spectra at higher concentrations. In view of these limitations and considering the results obtained by NMR spectroscopy, we simply estimated a value of $K_{D}>10^{4} \mathrm{M}^{-1}$ for the dimerization process. Our estimate is close but larger than the value reported by Jiang et al. using ${ }^{1} \mathrm{H}$ NMR spectroscopy.

The reasons why the aggregation of enantiomerically pure $\mathbf{Z n - 1}$ stops at the dimeric state are unknown for us. However, the analysis of the diffraction data of single crystals obtained from a $\mathrm{CHCl}_{3}$ solution of enantiopure $\mathbf{Z n - 1}$ previously described by Jiang and coworkers also revealed the exclusive presence of dimeric aggregates in the solid state [18]. Analogous results were obtained in the solid state for the enantiopure free base phthalocyanine $\mathbf{H}_{2}-\mathbf{1}$ [24].

Surprisingly, contrary to the sharp and well-defined signals observed in ${ }^{1} \mathrm{H}$ NMR spectra of enantiomerically pure samples of $(R)$ - and $(S)-\mathbf{Z n - 1}$ in $\mathrm{CDCl}_{3}$, an equimolar mixture of $(R)$ - and $(S)-\mathbf{Z n - 1}$ in $\mathrm{CDCl}_{3}$ solution, that is racemic Zn-1, did not produce observable proton signals when analyzed using ${ }^{1} \mathrm{H}$ NMR spectroscopy. This result suggested that in the racemic mixture the aggregation process does not stop at the dimeric state but continues with the formation of higher order colloidal aggregates that are not detectable by ${ }^{1} \mathrm{H}$ NMR spectroscopy [27].

\section{Insert Figure 2}

According to the magnitude of the estimated dimerization constant, the ${ }^{1} \mathrm{H}$ NMR spectrum of a $2 \mathrm{mM}$ solution of enantiopure $\mathbf{Z n - 1}$ displayed a number of proton signals in agreement with the $C_{4}$ symmetry of $[\mathbf{Z n - 1}]_{2}$ in a dimeric species. We performed a Diffusion-Ordered SpectroscopY (DOSY) experiment [28] using the above solution and determined a diffusion constant of $3.51 \pm 0.06 \times 10^{-10} \mathrm{~m}^{2} \cdot \mathrm{s}^{-1}$ for the $[\mathbf{Z n}-\mathbf{1}]_{2}$ dimer. Using the Stokes-Einstein equation we derived a hydrodynamic radius of $11.7 \AA$ for the $[\mathbf{Z n - 1}]_{2}$ dimer, which agrees well with the dimensions of the dimer estimated from simple molecular modelling studies (Figure S2).

The dimerization process of enantiopure $\mathbf{Z n - 1}$ was also investigated in solution by means of variable temperature ${ }^{1} \mathrm{H}$ NMR experiments (Figure 3). As stated above, at $298 \mathrm{~K}$, the ${ }^{1} \mathrm{H}$ NMR spectrum of a $2 \mathrm{mM}$ solution of enantiopure Zn1 in chloroform displayed two singlets at 9.30 and $7.83 \mathrm{ppm}$ corresponding to the chemically non-equivalent $\mathrm{H}_{\mathrm{a}}$ protons in the isoindole units of $\mathbf{Z n - 1}$ in the $[\mathbf{Z n - 1}]_{2}$ dimer (Figure 3). Lowering the temperature to $263 \mathrm{~K}$ resulted in the appearance of a new signal at $9.43 \mathrm{ppm}$ that was assigned to the chemically-equivalent isoindole $\mathrm{H}_{\mathrm{a}}$ protons of the monomer Zn-1. Further lowering of temperature induced an increase in the signal resonating at $9.43 \mathrm{ppm}$, corresponding to the monomer, at the expenses of those attributed to the $[\mathbf{Z n}-1]_{2}$ dimer. At $223 \mathrm{~K}$, the proton signals corresponding to the monomer were the only ones observable in the ${ }^{1} \mathrm{H}$ NMR spectrum. These results suggested that the monomer is favored at low temperatures while the dimer is favored on increasing the temperature. This was not an expected result; typically dimerization is favored at low temperatures. The thermodynamic signature for the dimerization process was obtained from a van't Hoff plot (Figure S4). The linear fit of the experimental data was good and allowed the calculation of the values of the thermodynamic contributions to the dimerization as $\Delta H=8.25$ kcal.mol ${ }^{-1}$ and $\Delta S=42.1$ cal.mol $^{-1} \cdot \mathrm{K}^{-1}$, corresponding to a $\Delta G=-4.28 \mathrm{kcal} . \mathrm{mol}^{-1}$ at $298 \mathrm{~K}$. The obtained thermodynamic parameters indicated that the dimerization process is endothermic and driven exclusively by entropy. This result may appear counterintuitive if one thinks that dimeric species are more ordered and both vibrationally and conformationally 
more constrained than the monomer. However, we can rationalize the obtained result by considering the solvation/desolvation processes that are involved with the dimerization equilibrium. The formation of the dimer $[\mathbf{Z n}-\mathbf{1}]_{2}$ is associated with a substantial reduction of the Pc's surface that is accessible to the solvent. In other words, upon dimerization a non-defined number of ordered solvent molecules that solvated one of the monomer's faces must be released to the bulk solution. The dimerization process definitively produces a gain in entropy when considering the solvent molecules. This large gain in entropy compensates the enthalpic cost of the dimerization process that is associated with a lower enthalpy for the $\pi-\pi$ interactions of the dimer than for the solvated monomers. When the temperature is increased the released solvent molecules pay for the energetic cost of the dimerization process which is enthalpically unfavorable. This is translated in the observed increase of the dimerization equilibrium constant at higher temperatures. Entropy-driven dimerization processes in organic solvents are uncommon but have been observed for other systems such as Cram's velcrands [29] and Rebek's molecular capsules [30].

\section{Insert Figure 3}

\section{Solution studies of quinuclidine binding to $\mathrm{Zn}-1$}

The binding of quinuclidine $\mathbf{2}$ to enantiopure $\mathbf{Z n - 1}$ was studied by means of UV-vis and ${ }^{1} \mathrm{H}$ NMR spectroscopy. The concentration of $\mathbf{Z n - 1}$ used to perform the binding studies plays an important role in determining its distribution into monomeric and dimeric species [31]. The coordination of quinuclidine to enantiopure $\mathbf{Z n - 1}$ was first probed by UV-vis titration experiments using a $4 \times 10^{-6} \mathrm{M}$ chloroform solution of $\mathbf{Z n - 1}$. From the dimerization studies, one can derive that under these diluted conditions Zn-1 exists in solution exclusively as a monomer (Figure S3). The addition of increasing amounts of quinuclidine to a $4 \mu \mathrm{M}$ solution of enantiopure $\mathbf{Z n - 1}$ resulted in a gradual diminution of the Soret band centered at $355 \mathrm{~nm}$ and the concomitant appearance of a red-shifted band with maximum at $362 \mathrm{~nm}$ (Figure 4). This 7 $\mathrm{nm}$ red-shift is associated with the axial coordination of the quinuclidine ligand to the $\mathrm{Zn}$ center of $\mathbf{Z n - 1}$ to form a $1: 1$ complex, as typically observed for Zn-porphyrins [32]. Moreover, the titration spectra provided a clear isosbestic point centered at $361 \mathrm{~nm}$. This observation suggested the presence of a single equilibrium involving two colored species in solution. Based on previously reported coordination studies using Zn-porphyrins, we assigned the two species to free Zn-1 and the 1:1 complex $\mathbf{2} \bullet \mathbf{Z n}-\mathbf{1}$ having one molecule of quinuclidine axially coordinated to the zinc metal center. The analysis of the experimental titration data using a theoretical 1:1 binding isotherm gave a good fit and returned an association constant value of $K(\mathbf{2} \bullet \mathbf{Z n}-\mathbf{1})=1.69 \times 10^{6} \mathrm{M}^{-1}$ for the $\mathbf{2} \bullet \mathbf{Z n} \mathbf{- 1}$ complex (Figure 4). This value is two orders of magnitude larger than the one measured for coordination complexes of quinuclidine with zinc-porphyrins [33]. Most likely, the Lewis acidity of the zinc(II) metal center is increased in the Pc derivatives compared to their porphyrin analogues provoking the strengthening of the coordination bonds.

\section{Insert Figure 4}

When the binding process of quinuclidine with enantiopure $\mathbf{Z n - 1}$ was studied using ${ }^{1} \mathrm{H}$ NMR spectroscopy complexes of larger stoichiometry than the simple 1:1 were detected. At NMR concentration ([Zn-1] $\left.2 \times 10^{-3} \mathrm{M}\right)$ and room temperature, enantiopure $\mathbf{Z n - 1}$ is exclusively present in solution as the dimer $[\mathbf{Z n}-\mathbf{1}]_{2}$ (Figure 5 a). The addition of 0.3 equiv of quinuclidine to a $2 \mathrm{mM} \mathrm{CDCl}_{3}$ solution of $\mathbf{Z n - 1}$ resulted in the emergence of a new singlet $\left(\mathrm{H}_{\mathrm{a}^{\prime}}\right)$ in the downfield region of the ${ }^{1} \mathrm{H}$ NMR spectrum (Figure 5b). This new singlet emerging at 9.13 ppm was attributed to isoindole aromatic protons of Zn-1 in a coordination complex of unknown stoichiometry. Concomitantly, three new signals appeared in the upfield region of the ${ }^{1} \mathrm{H}$ NMR spectrum. These signals resonated at $-2.85,-0.7$, and $0 \mathrm{ppm}$ and were assigned to the $\mathrm{H}_{1}, \mathrm{H}_{2}$ ' and $\mathrm{H}_{3}$ ' protons of bound quinuclidine. The notable upfield shift experienced by the quinuclidine protons compared to their chemical shifts in the free ligand $\left(\Delta \delta=-5.7,-2.2\right.$ and $-1.7 \mathrm{ppm}$ for the $\mathrm{H}_{1}, \mathrm{H}_{2}$ 
and $\mathrm{H}_{3}$, respectively), pointed to the formation of an axially coordinated complex between quinuclidine and $\mathbf{Z n - 1}$. The protons of the bound quinuclidine experienced a large upfield shift induced by the ring-current that was more evident for the $\alpha$-quinuclidine protons $\left(\mathrm{H}_{1}{ }^{\prime}\right)$ due to its close proximity to the $\mathrm{Pc} \pi$-system.

\section{Insert Figure 5}

Taken together, these observations suggested the initial formation of a $2: 1$ complex $\mathbf{2} \bullet[\mathbf{Z n}-\mathbf{1}]_{\mathbf{2}}$, in which one quinuclidine molecule is bound to one of two faces of the [ $\mathbf{Z n - 1 2}$ ] dimer. Addition of incremental amounts of 2 (up to 0.5 equiv) provoked a decrease in the intensity of the proton signals assigned to the initially formed 2:1 complex. Simultaneously, a new broad signal resonating at $9.29 \mathrm{ppm}\left(\mathrm{H}_{\mathrm{a}}{ }^{\prime}{ }^{\prime}\right)$ emerged and a new set of three signals for bound quinuclidine, resonating at $-3.06,-0.83$ and $-0.08 \mathrm{ppm}$, and assigned as $\mathrm{H}_{1}{ }^{\prime}, \mathrm{H}_{2}{ }^{\prime}$ ' and $\mathrm{H}_{3}{ }^{\prime}$, appeared (Figure 5c). We attributed this new set of signals to the formation of a $2: 2$ complex $\mathbf{2}_{2} \bullet[\mathbf{Z n}-\mathbf{1}]_{\mathbf{2}}$ having one quinuclidine axially coordinated in each one of the two faces of the $\left[\mathbf{Z n - 1} \mathbf{1}_{2}\right]$ dimer. A further increase in the quinuclidine concentration gradually shifted the equilibrium towards the preferential formation of the 2:2 complex. Nonetheless, in the presence of 0.7 equiv of quinuclidine (Figure $5 \mathrm{~d}$ ) a third set of signals for the bound ligand emerged (-1.6, -0.1 and $0.4 \mathrm{ppm}, \mathrm{H}_{1}{ }^{\prime}$, , $\mathrm{H}_{2}$ "' and $\mathrm{H}_{3}$ ', ', respectively) in the upfield region of the spectrum together with a new broad aromatic signal resonating at $9.43 \mathrm{ppm}\left(\mathrm{H}_{\mathrm{a}}{ }^{\prime \prime}\right)$. The downfield shift of this aromatic signal suggested that the newly formed complex should have a 1:1 stoichiometry $(\mathbf{2} \bullet \mathbf{Z n}-\mathbf{1})$. We have observed an analogous downfield shift of the isoindole protons in the Zn-1 monomer. The emergent $\mathbf{2 \bullet Z n - 1}$ complex will result from the dissociation of the previously formed 2:2 complex, whose concentration had increased (Figure 6). The simulated speciation profile for the quinuclidine binding to enantiopure $\mathbf{Z n - 1}$ at millimolar concentration, considering dimerization constants for $\mathbf{Z n - 1}$ and $\mathbf{2} \bullet \mathbf{Z n - 1}$ as $\mathrm{K}_{\mathrm{D}}=10^{4} \mathbf{M}^{-1}$ and $\mathrm{K}_{\mathrm{D}}=0.6 \times 10^{3} \mathrm{M}^{-1}$ (vide infra), respectively, and a microscopic binding constant value of $K=1.69 \times 10^{6} \mathrm{M}^{-1}$ for the interaction of quinuclidine with enantiopure $\mathbf{Z n - 1}$ in any of its stoichiometric states, was in complete agreement with the results obtained in the above ${ }^{1} \mathrm{H}$ NMR titration (Figure S5).

\section{Insert Figure 6}

The addition of more than 1 equiv of quinuclidine to the solution, provoked the selective disappearance of the quinuclidine signals assigned to the 1:1 complex but not to those of the parent 2:2 complex (Figure 5f). In contrast, the proton signals corresponding to the isoindole units in the $1: 1$ and the 2:2 complex did not experience noticeable changes. These observations together with the lack of well-defined proton signals in the ${ }^{1} \mathrm{H}$ NMR spectra of mixture for free quinuclidine added in excess, suggested the existence of a chemical exchange process that was intermediate on the chemical shift timescale between the $1: 1, \mathbf{2} \bullet \mathbf{Z n}-\mathbf{1}$, complex and free quinuclidine. The differences in the exchange kinetics observed for free quinuclidine and bound quinuclidine in the $1: 1, \mathbf{2} \bullet \mathbf{Z n - 1}$, (intermediate) or the $2: 2, \mathbf{2}_{2} \bullet[\mathbf{Z n}-\mathbf{1}]_{\mathbf{2}}$, (slow) complexes suggested that they were involved in exchange mechanisms with different energy barriers (Figure S6). We hypothesize that in the 2:2 complex the exchange of bound quinuclidine implies the complete cleavage of the $\mathrm{Zn}(\mathrm{II}) \cdots \mathrm{N}$ coordination bond prior to the exchange with the free ligand (i.e. "dissociative-like" ligand exchange mechanism). Conversely, in a 1:1 complex the face opposite to the bound ligand is available for the entrance of the exchange partner. Most likely, the approach of the incoming quinuclidine occurs synchronously to the departure of the one leaving. Thus, the ligand exchange occurs through an "associative-like" mechanism. The energy barrier for the "dissociative-like" exchange (2:2 complex) should be higher compared to the "associative-like" one (1:1) because the former requires the complete cleavage of the coordination bond prior to the exchange. This translates into the slow exchange process on the ${ }^{1} \mathrm{H}$ NMR timescale observed for the free/bound quinuclidine in the $2: 2, \mathbf{2}_{2} \bullet[\mathbf{Z n}-\mathbf{1}]_{2}$, complex. Likewise, the lower energy barrier that might be associated with the ligand exchange in the $1: 1, \mathbf{2 \bullet Z n - 1}$, complex 
would explain the intermediate rate on the ${ }^{1} \mathrm{H}$ NMR timescale observed for exchange process of the free/bound quinuclidine in this complex.

In order to reduce the exchange rate of quinuclidine in the $1: 1, \mathbf{2} \cdot \mathbf{Z n} \mathbf{- 1}$, complex we performed variable temperature ${ }^{1} \mathrm{H}$ NMR experiments using a $2 \mathrm{mM} \mathrm{CDCl}_{3}$ solution of $\mathbf{Z n - 1}$ in the presence of a large excess of quinuclidine (Figure S7). Unfortunately, we were not able to observe separate proton signals for free and bound quinuclidine in the solution containing the $1: 1, \mathbf{2 \bullet Z n - 1 , ~ c o m p l e x ~ e v e n ~ a t ~} 213 \mathrm{~K}$. The only significant change we observed in the v.t. ${ }^{1} \mathrm{H}$ NMR spectra was the decrease in intensity of the proton signals corresponding to the $2: 2, \mathbf{2}_{\mathbf{2}} \bullet\left[\mathbf{Z n} \mathbf{- 1} \mathbf{2}_{\mathbf{2}}\right.$, complex and the concomitant increase of the ones corresponding to the 1:1 complex. This observation indicated that at lower temperatures the $1: 1 \leftrightarrow 2: 2$ equilibrium was shifted towards the formation of the $1: 1, \mathbf{2 \bullet Z n - 1}$, complex whereas at higher temperatures the $\pi$-aggregation was favored. This result was in complete agreement with the previous findings made for the v.t. study of the dimerization process of $\mathbf{Z n - 1}$.

We observed that at room temperature the ${ }^{1} \mathrm{H}$ NMR spectrum of a $\mathrm{CDCl}_{3}$ solution containing $2 \mathrm{mM}$ enantiopure $\mathbf{Z n}$ 1 and 3 equiv of 2 showed proton signals for dimeric and monomeric coordinated species (2:2 and 1:1 complexes, respectively). This observation indicated a reduction in the dimerization affinity for the coordinated monomer compared to the non-coordinated counterpart. Using the values of the integral areas of the most downfield shifted proton signals of the $\mathbf{2 \bullet Z n - 1}$ and $[\mathbf{2} \bullet \mathbf{Z n}-\mathbf{1}]_{2}$ complexes we calculated a dimerization constant value of $K_{\mathrm{D}}\left([2 \bullet \mathrm{Zn}-1]_{2}\right)=0.6 \times 10^{3} \mathrm{M}^{-1}$ at $298 \mathrm{~K}$ for the enantiopure $\mathbf{2}_{2} \bullet[\mathbf{Z n}-\mathbf{1}]_{\mathbf{2}}$ complex. This value is indeed lower than our estimate for the dimerization constant of free Zn-1 $\left(K_{\mathrm{D}}>10^{4} \mathrm{M}^{-1}\right.$, vide supra $)$ and Jiang's reported value $\left(K_{D}=7.3 \times 10^{3} \mathrm{M}^{-1}\right)$. The strength of $\pi-\pi$ interactions responsible for the dimer formation is reduced upon coordination of the axial ligand. Probably, the complexation of quinuclidine to the $\mathrm{Zn}$ (II) metal center of the $\mathbf{Z n - 1}$ Pc distorted its planarity resulting in weaker $\pi$ - $\pi$ interactions between the two coordinated monomers in the dimer [34]. We also determined thermodynamic quantities of the dimerization process for the enantiopure and axially coordinated $\mathbf{2} \bullet \mathbf{Z n - 1}$ complex as $\Delta H=7.90 \mathrm{kcal}_{\text {. }} \mathrm{mol}^{-1}$ and $\Delta S=$ 39.41 cal. $\mathrm{mol}^{-1} . \mathrm{K}^{-1}$ using a van't Hoff plot. Not surprisingly, these values are very similar to the ones determined for the non-coordinated Zn-1 counterpart (Figure S8).

As expected, separated $\mathrm{CDCl}_{3}$ solutions of enantiomerically pure complexes $(R)$ and $(S)$-[Zn-1] containing an excess of quinuclidine ligand ( 3 equiv) displayed identical ${ }^{1} \mathrm{H}$ NMR spectra that showed the diagnostic proton signals for the chiral monomer and the corresponding chiral dimer. Remarkably, the ${ }^{1} \mathrm{H}$ NMR spectrum of a solution containing racemic Zn-1 and 3 equiv of $\mathbf{2}$ displayed an additional set of proton signals. This was especially visible in the upfield region of the spectrum (Figure $7 \mathrm{~b}$ ) and we assigned the new set of signals to quinuclidine protons in the meso-dimer $\mathbf{2}_{2} \bullet[(R, S)-\mathbf{Z n}-\mathbf{1}]_{2}$. Simple integration of the signals corresponding to the homo $\left(\mathbf{2}_{2} \bullet[(R, R)-\mathbf{Z n}-\mathbf{1}]_{2}, \mathbf{2}_{2} \bullet[(R, R)-\mathbf{Z n} \mathbf{- 1}]_{2}\right)$ and hetero dimeric complexes $\left(\mathbf{2}_{2} \bullet[(R, S)-\mathbf{Z n}-\mathbf{1}]_{2}\right)$ allowed the calculation of an equilibrium constant value for the selfsorting process of the chiral homodimers into the meso-heterodimer as $K_{\mathrm{ss}}=4$. This value coincides with the statistical estimate indicating that all dimers are isoenergetic and the self-sorting process of racemic $\mathbf{2} \bullet \mathbf{Z n} \mathbf{- 1}$ complexes into dimers does not show signs of chiral discrimination (self-recognition or self-discrimination).

\section{Insert Figure 7}

\section{EXPERIMENTAL}

\section{General}


${ }^{1} \mathrm{H}$ NMR spectra were recorded on a Bruker Avance 400 (400 MHz for ${ }^{1} \mathrm{H}$ NMR spectroscopy), or a Bruker Avance 500 (500 MHz for ${ }^{1} \mathrm{H}$ NMR spectroscopy) ultrashield spectrometer. The deuterated solvents (Aldrich) used are indicated and chemical shifts are given in ppm. For $\mathrm{CDCl}_{3}$, the peaks were referenced relative to the solvent residual peak $\delta_{\mathrm{H}}=7.26 \mathrm{ppm}$. High-resolution mass spectra were obtained using a Bruker Autoflex MALDI-TOF mass spectrometer. Thin-layer chromatography (TLC) and flash column chromatography were performed with DC-aufolien Kieselgel $60 \mathrm{~F}_{254}$ (Merck) and Scharlab 60 silica gel, respectively. UV-vis measurements were carried out using a Shimadzu UV-2410PC spectrophotometer equipped with a photomultiplier detector, double-beam optics, and D2 and W light sources and using a $1 \mathrm{~mm}$ path cell for the dimerization studies.

\section{Materials}

All commercial solvents and chemicals were of reagent grade quality and were used without further purification. Dry DMF was acquired form a Solvent Purification System MBraun SPS-800. Deuterated chloroform and chloroform were deacidified by passing through a short column of aluminum oxide 90 active, neutral (Merck).

\section{Synthesis}

\section{Preparation of (R)- and (S)-phthalocyanato zinc (Zn-1)}

A mixture of enantiomerically pure $(\mathrm{R})$ or $(\mathrm{S})$-benzo[b]dinaphtho[2,1-e:1',2'-g][1,4]dioxocine-5,6-dicarbonitrile (200 mg, $0.489 \mathrm{mmol}), \mathrm{Zn}(\mathrm{OAc})_{2} \cdot 2 \mathrm{H}_{2} \mathrm{O}(22.4 \mathrm{mg}, 0.122 \mathrm{mmol})$ and DBU $(0.05 \mathrm{~mL})$ were refluxed at $160{ }^{\circ} \mathrm{C}$ in $1-$ hexanol $(2 \mathrm{~mL})$ for $4 \mathrm{~h}$. After reaction time the mixture was cooled at room temperature, the volatiles were removed under reduced pressure and the crude was purified by silica gel column chromatography using $\mathrm{CH}_{2} \mathrm{Cl}_{2}$ : $\mathrm{THF}(100: 2)$ as eluent mixture. The solvent of the collected fractions was evaporated in vacuum to yield $52 \mathrm{mg}$ of the (R)-enantiomer as a green powder (25\%). ${ }^{1} \mathrm{H} \mathrm{NMR}\left(400 \mathrm{MHz} ; \mathrm{CDCl}_{3} ; \mathrm{Me}_{4} \mathrm{Si}\right): \delta_{\mathrm{H}}, \mathrm{ppm}: 6.84(1 \mathrm{H}, \mathrm{d}, J=8.46 \mathrm{~Hz}), 6.96(1 \mathrm{H}, \mathrm{t}, J=7.58$ $\mathrm{Hz}), 7.11(1 \mathrm{H}, \mathrm{d}, J=8.46 \mathrm{~Hz}), 7.20(1 \mathrm{H}, \mathrm{t}, J=7.58), 7.32(1 \mathrm{H}, \mathrm{t}, J=7.58 \mathrm{~Hz}), 7.44(1 \mathrm{H}, \mathrm{t}, J=7.58 \mathrm{~Hz}), 7.50(1 \mathrm{H}, \mathrm{d}, J$ $=8.46 \mathrm{~Hz}), 7.69(1 \mathrm{H}, \mathrm{d}, J=8.46 \mathrm{~Hz}), 7.83(1 \mathrm{H}, \mathrm{s}), 7.87(1 \mathrm{H}, \mathrm{d}, \mathrm{J}=8.46 \mathrm{~Hz}), 7.97(1 \mathrm{H}, \mathrm{d}, \mathrm{J}=8.46), 8.46(1 \mathrm{H}, \mathrm{d}, 8.46$ $\mathrm{Hz}), 8.66(1 \mathrm{H}, \mathrm{d}, 8.46 \mathrm{~Hz}), 9.30(1 \mathrm{H}, \mathrm{s})$. HRMS (MALDI+): $1704.3527 \mathrm{~m} / z$ (calcd. for [M] $\left.{ }^{+} 1704.3513\right)$. ${ }^{1} \mathrm{H}$ NMR data are in complete agreement with those reported in the literature [18].

\section{Titrations and data analysis}

${ }^{1} \mathrm{H}$ NMR and UV-vis titrations were performed by addition of incremental amounts of a solution containing the ligand to a solution of the $\mathbf{Z n - 1}$ in either a $5 \mathrm{~mm}$ NMR tube or a $1 \mathrm{~cm}$ path quartz cuvette by using microliter syringes. After each addition the sample mixture was hand shacked and the spectrum recorded. In both types of titration experiments, Zn-1 was present in the ligand solution at the same concentration than that in the NMR tube or cuvette to avoid dilution effects. Deacidified chloroform and deacidified deuterated chloroform were used as solvent for the UVvis and ${ }^{1} \mathrm{H}$ NMR titration, respectively. UV-vis spectrophotometric titrations were analyzed by fitting the whole series of spectra at $1 \mathrm{~nm}$ intervals by using the software ReactLab Equilibria 1.1 from Jplus Consulting Pty Ltd (8 Windsor Road, East Fremantle, WA 6158, Australia).

\section{CONCLUSIONS}

The dimerization process of enantiopure Zn-1 was studied using UV-vis dilution experiments and variable temperature ${ }^{1} \mathrm{H}$ NMR experiments. Unexpectedly, we found that the dimerization process was favored at high temperatures. The calculated thermodynamic contributions of the dimerization process revealed that it is entropy-driven 
and enthalpically opposed. We rationalized these results considering the solvation/desolvation processes that are involved in the dimerization equilibria.

The coordination of quinuclidine $\mathbf{2}$ to enantiopure $\mathbf{Z n - 1}$ was studied at two different concentrations using UV-vis and ${ }^{1} \mathrm{H}$ NMR titrations. At micromolar concentration, enantiopure $\mathbf{Z n - 1}$ is exclusively present in solution as a monomer and formed simple $1: \mathbf{1}, \mathbf{2} \bullet \mathbf{Z n} \mathbf{- 1}$, complexes. The calculated association constant for the $\mathbf{2} \bullet \mathbf{Z n - 1}$ complex is larger than $10^{6}$ $\mathrm{M}^{-1}$. This value is two orders of magnitude higher than the ones typically reported for the coordination of 2 with $\mathrm{Zn}$ porphyrins. At millimolar concentration, enantiopure $\mathbf{Z n - 1}$ is present in solution mainly as a dimer. The incremental addition of quinuclidine to $\mathrm{mM}$ solutions of enantiopure $\mathbf{Z n - 1}$, produced quinuclidine:Zn-1 complexes of larger stoichiometry than the simple 1:1. Using ${ }^{1} \mathrm{H}$ NMR spectroscopy, it was possible to demonstrate the formation of $1: 2$ $\left(\mathbf{2} \bullet[\mathbf{Z n}-\mathbf{1}]_{\mathbf{2}}\right)$ and 2:2 $\left(\mathbf{2}_{2} \bullet[\mathbf{Z n}-\mathbf{1}]_{\mathbf{2}}\right)$ complexes in addition to the known 1:1 (2•Zn-1) complex. We measured a reduction in the dimerization constant value of the coordinated enantiopure monomer $\mathbf{2} \cdot \mathbf{Z n} \mathbf{- 1}$ compared to the parent uncoordinated metallo-phthalocyanine Zn-1. Most likely, the axial coordination of quinuclidine to the $\mathrm{Zn}$ (II) metal centers bows the planarity of the Pc weakening the $\pi-\pi$ interactions responsible for dimer $\mathbf{2}_{2} \bullet[\mathbf{Z n} \mathbf{- 1}]_{\mathbf{2}}$ formation. The addition of an excess of quinuclidine to a millimolar solution of enantiopure $\mathbf{Z n - 1}$ induced the selective disappearance of the quinuclidine signals assigned to the 1:1 complex. The protons of the quinuclidine in the 2:2 complex remained unaltered. This observation prompted us to propose a different exchange mechanism between free and bound quinuclidine in the two complexes. The dissimilar kinetics observed on the NMR chemical shift timescale for the two exchange processes are related to different energy barriers for them (dissociative exchange and associative exchange).

Finally, we demonstrated that the dimerization of racemic Zn-1 (equimolar mixture of $(R)$ - and $(S)$-Zn-1) produced large oligomers. Remarkably, when the dimerization study of racemic Zn-1 was performed in a solution containing an excess of quinuclidine, we observed the assembly of both the chiral homodimers and the meso-heterodimer. The ratio of the dimers coincided with the expected statistical estimate assuming isoenergetic species, indicating a lack of chiral discrimination in the self-sorting process of racemic $(R, S)-\mathbf{2} \bullet \mathbf{Z n}-\mathbf{1}$ into dimers

\section{ACKNOWLEDGEMENTS}

The authors thank the European Union (ERC Stg grant 279313, CHEMCOMP), Gobierno de España MINECO (projects CTQ2014-56295-R, CTQ2014-52974-REDC and Severo Ochoa Excellence Accreditation 2014-2018 SEV2013-0319), FEDER funds (project CTQ2014-56295-R) and the ICIQ Foundation for funding.

\section{REFERENCES}

1 Tong WY, Djurisic AB, Xie MH, Ng ACM, Cheung KY, Chan WK, Leung YH, Lin HW and Gwo S. J. Phys. Chem. B 2006; 110: 17406-17413.

2 Duzhko V and Singer KD. J. Phys. Chem. C. 2007; 111: 27-31.

3 Guillaud G, Simon J and Germain JP. Coord. Chem. Rev. 1998; 178: 1433-1484.

4 Ishikawa N, Sugita M, Ishikawa T, Koshihara S and Kaizu Y. J. Am. Chem. Soc. 2003; 125: 8694-8695.

5 Loi MA, Denk P, Hoppe H, Neugebauer H, Winder C, Meissner D, Brabec C, Sariciftci NS, Gouloumis A, Vazquez P and Torres T. J. Mater. Chem. 2003; 13: 700-704. 
6 Hofman JW, van Zeeland F, Turker S, Talsma H, Lambrechts SAG, Sakharov DV, Hennink WE and van Nostrum CF. J. Med. Chem. 2007; 50: 1485-1494.

7 Kolarova H, Nevrelova P, Bajgar R, Hrova D, Kejlova K and Strnad M. Toxicol. in Vitro 2007; 21: 249-253.

8 de la Torre G, Vazquez P, Agullo-Lopez F and Torres T. J. Mater. Chem. 1998; 8: 1671-1683.

9 Nemykin VN and Lukyanets EA. Arkivoc 2010: 136-208.

10 Kobayashi N. Coord. Chem. Rev. 2001; 219: 99-123.

11 Kimura M, Kuroda T, Ohta K, Hanabusa K, Shirai H and Kobayashi N. Langmuir 2003; 19: 4825-4830.

12 Hembury GA, Borovkov VV and Inoue Y. Chem. Rev. 2008; 108: 1-73.

13 Furusho Y, Kimura T, Mizuno Y and Aida T. J. Am. Chem. Soc. 1997; 119: 5267-5268.

14 Lu HJ and Zhang XP. Chem. Soc. Rev. 2011; 40: 1899-1909.

15 Vannostrum CF, Bosman AW, Gelinck GH, Picken SJ, Schouten PG, Warman JM, Schouten AJ and Nolte RJM. J. Chem. Soc., Chem. Commun. 1993: 1120-1122.

16 Kobayashi N, Kobayashi Y and Osa T. J. Am. Chem. Soc. 1993; 115: 10994-10995.

17 Kobayashi N, Narita F, Ishii K and Muranaka A. Chem-Eur. J. 2009; 15: 10173-10181.

18 Wang K, Qi DD, Wang HL, Cao W, Li WJ and Jiang JZ. Chem-Eur. J. 2012; 18: 15948-15952.

19 Hunter CA, Meah MN and Sanders JKM. J. Am. Chem. Soc. 1990; 112: 5773-5780.

20 Taylor PN and Anderson HL. J. Am. Chem. Soc. 1999; 121: 11538-11545.

21 Liu CHJ and Lu WC. J. Chin. Inst. Chem. Eng. 2007; 38: 483-488.

22 Przybyl B and Janczak J. Dyes Pigm. 2014; 100: 247-254.

23 D'Souza F and Ito O. Chem. Commun. 2009: 4913-4928.

24 Wang K, Wang HL, Mack J, Li WJ, Kobayashi N and Jiang JZ. Acta Chim. Sinica. 2012; 70: 1791-1797.

25 The absolute configuration of all the BINOL units is the same, all- $(R)-\mathbf{Z n - 1}$ or all- $(S)-\mathbf{Z n}-\mathbf{1}$.

26 The chemical exchange of the monomers within the dimeric species is slow on the ${ }^{1} \mathrm{H}$ NMR timescale.

27 Dynamic Light Scattering (DLS) experiments on $0.1 \mathrm{mM} \mathrm{CHCl}_{3}$ solutions of enantiomerically pure (S)-Zn-1 and of an equimolar mixture of $(R)$ - and $(S)$-Zn-1 gave an average radii of $1.71 \mathrm{~nm}$ and $337.4 \mathrm{~nm}$, respectively. These results demonstrated that in the racemic mixture of $(R)$ - and $(S)-\mathbf{Z n - 1}$ aggregates of bigger dimensions are formed compared to the enantiomerically pure solutions.

28 Johnson CS. Prog. Nucl. Magn. Reson. Spectrosc. 1999; 34: 203-256.

${ }_{29}$ Cram DJ, Choi HJ, Bryant JA and Knobler CB. J. Am. Chem. Soc. 1992; 114: 7748-7765.

30 Kang JM and Rebek J. Nature 1996; 382: 239-241.

${ }_{31}$ Chi XL, Guerin AJ, Haycock RA, Hunter CA and Sarson LD. J. Chem. Soc., Chem. Commun. 1995: 2567-2569.

32 Hunter CA, Sanders JKM and Stone AJ. Chem. Phys. 1989; 133: 395-404.

33 Ballester P, Costa A, Castilla AM, Deya PM, Frontera A, Gomila RM and Hunter CA. Chem-Eur. J. 2005; 11: 21962206.

34 Andryushkevich SO, Birin KP, Gorbunova YG and Tsivadze AY. Prot. Met. Phys. Chem. Surf., 2011; 47: 494-502. 


\section{FIGURE CAPTIONS}

Figure 1. Dimerization equilibrium of the chiral the chiral zinc phthalocyanine $\mathbf{Z n - 1}$. The structure of $\mathbf{Z n - 1}$ monomer is shown as line-drawing structure and the dimer $[\mathbf{Z n - 1}]_{2}$ as MM3 energy minimized structure (protons are omitted for clarity).

Figure 2. UV-Vis spectra of $\mathbf{Z n - 1}$ at different concentrations $\left(1 \times 10^{-5} \mathrm{M}\right.$ to $\left.1.24 \times 10^{-4} \mathrm{M}\right)$ in chloroform recorded during the dilution experiment.

Figure 3. Left: Selected region of the variable temperature ${ }^{1} \mathrm{H}$ NMR spectra of a $2 \mathrm{mM}$ chloroform solution of $\mathbf{Z n - 1}$ showing the proton signal corresponding to the isoindole unit of the monomer (M) and one of the two isoindole protons displayed by the dimer (D). Right: MM3 energy minimized structures of the monomeric and dimeric species of $\mathbf{Z n - 1}$.

Figure 4. MM3 energy minimized structures representing the quinuclidine coordination to the Zn-1 monomer (top) and UV-vis titration spectra (Soret region) of $\mathbf{Z n - 1}$ with increasing amounts of quinuclidine in chloroform at 298K (from 0 to 7 eq) (bottom). The concentration of the zincphthalocyanine was maintained constant throughout the titration $\left(4 \times 10^{-6} \mathrm{M}\right)$. Inset: Inset: Fit of the experimental data at 355 and 365 nm to the calculated theoretical 1:1 binding isotherm.

Figure 5. Selected regions of the ${ }^{1} \mathrm{H}$ NMR spectrum recorded during a titration of $\mathbf{Z n - 1}$ with increasing amounts of quinuclidine $\mathbf{2}$ displaying the progressive formation of the 2:1, 2:2 and 1:1 complexes. ([Zn-1] $=2 \cdot 10^{-3} \mathrm{M}, \mathrm{CDCl}_{3}, 400 \mathrm{MHz}$ ). Primed letter corresponded to $1: 2$ complex $\mathbf{2} \cdot[\mathbf{Z n}-$ $\mathbf{1}]_{2}$, doubly primed to the $2: 2$ complex $\mathbf{2}_{2} \bullet[\mathbf{Z n}-\mathbf{1}]_{2}$, and triply primed to the $1: 1$ complex $\mathbf{2} \cdot[\mathbf{Z n}-\mathbf{1}]$.

Figure 6. Binding model used to rationalize the binding of quinuclidine $\mathbf{2}$ to the $[\mathbf{Z n}-\mathbf{1}]_{\mathbf{2}}$. MM3 energy minimized structures are shown for the species involved.

Figure 7. a) Selected regions of the ${ }^{1} \mathrm{H}$ NMR spectrum of $(R)-\mathbf{Z n}-\mathbf{1}$ with 3 equiv of quinuclidine and b) Selected regions of the ${ }^{1} \mathrm{H}$ NMR spectrum of the equimolar mixture of $(R)$ and $(S)-\mathbf{Z n - 1}$ with 3 equiv of quinuclidine. 

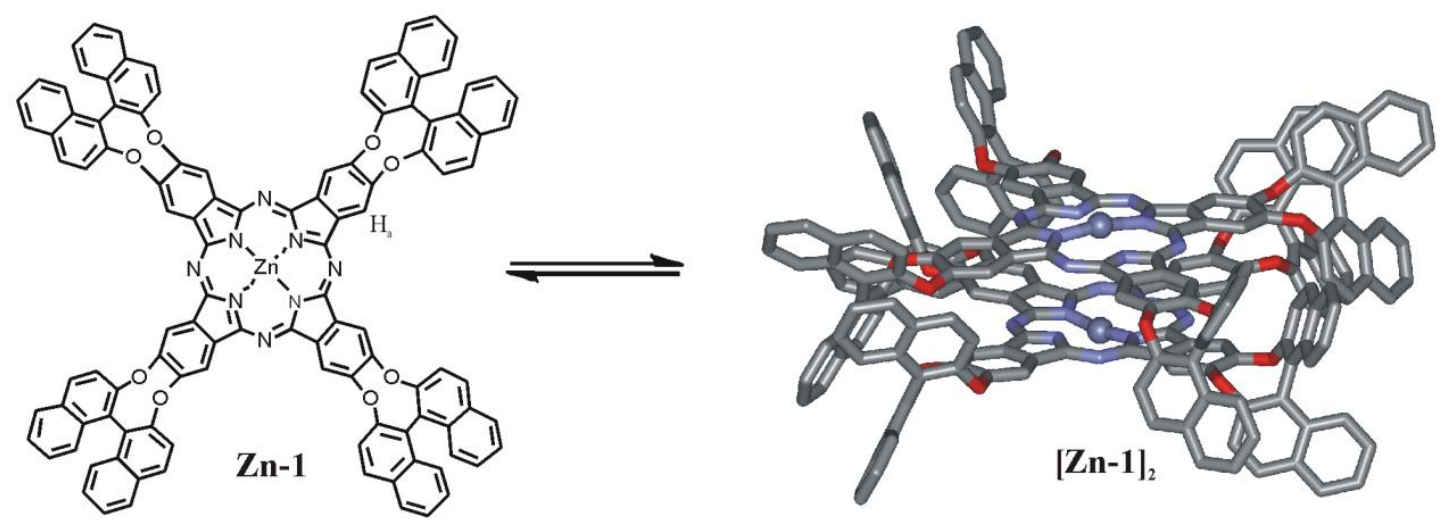

Figure 1 


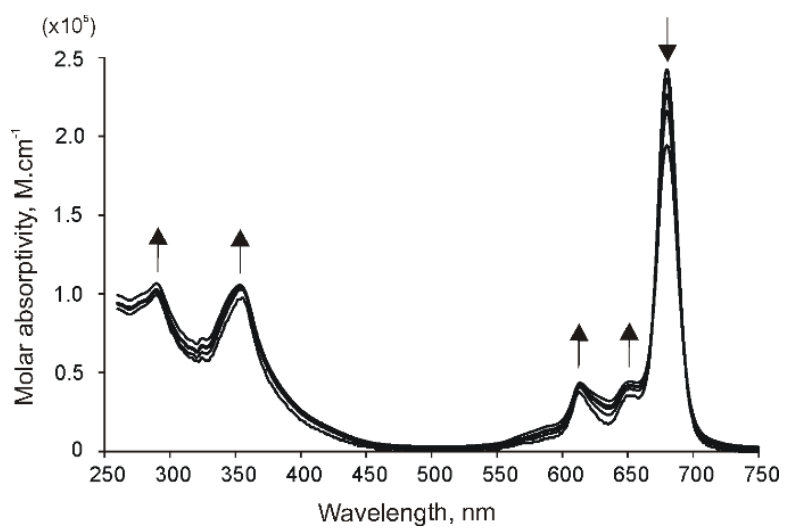

Figure 2 

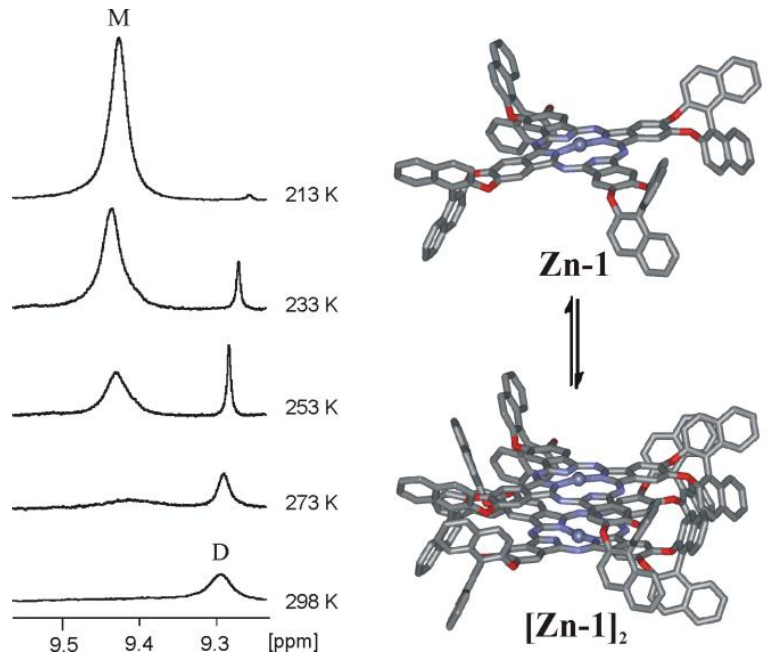

Figure 3 

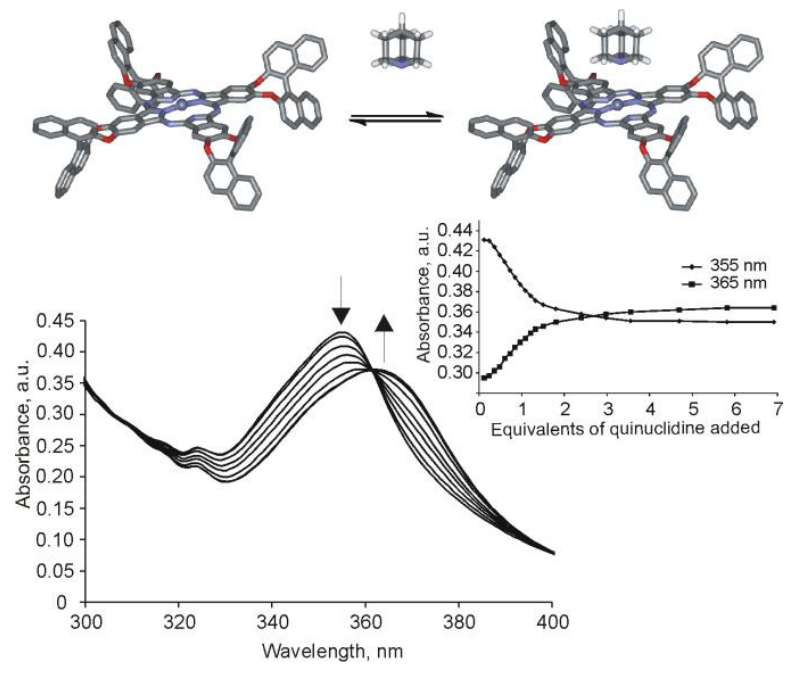

Figure 4 

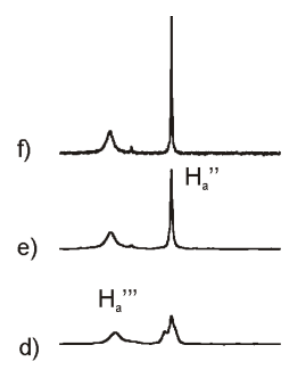

c)

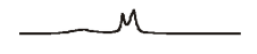

b)

a) $\overbrace{\frac{1}{9.5}, T_{9.0}}^{\mathrm{H}_{\mathrm{a}}}$

Figure 5

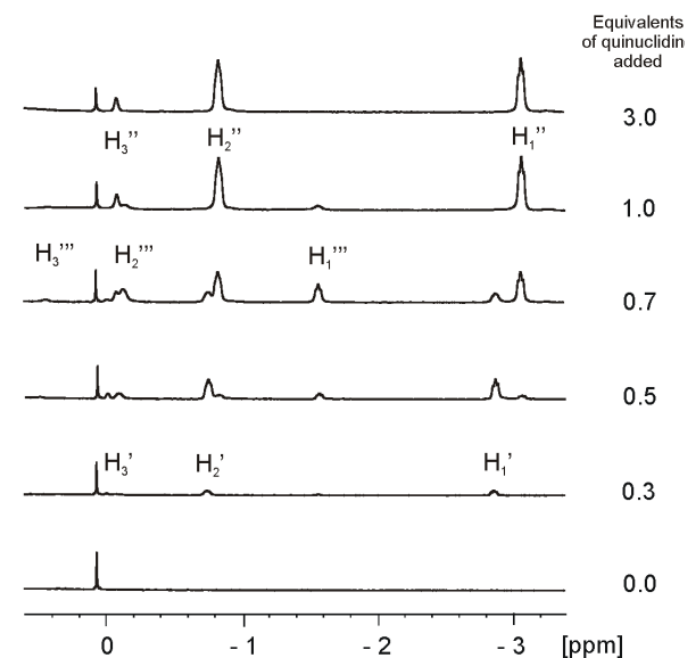

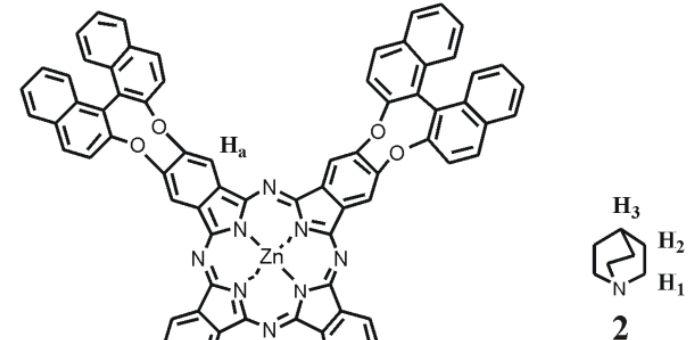




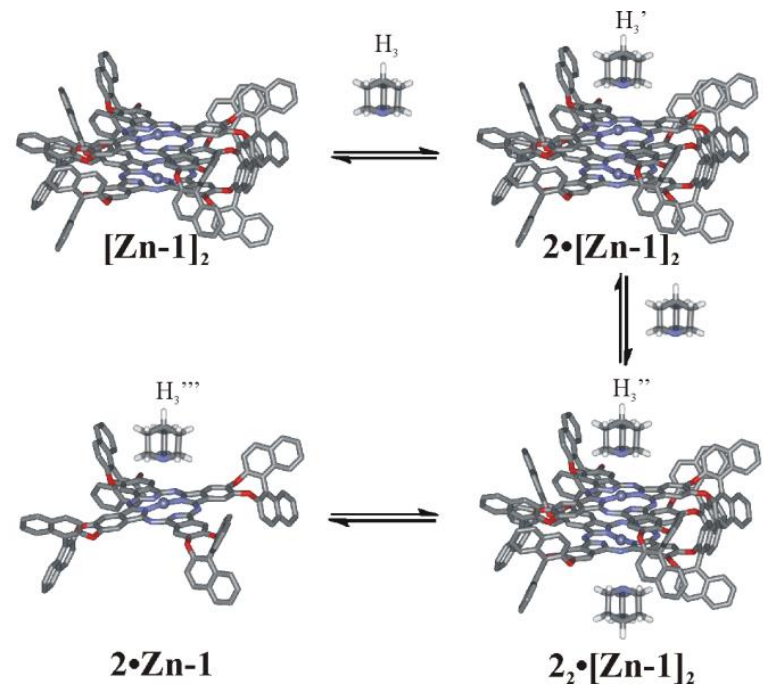

Figure 6 


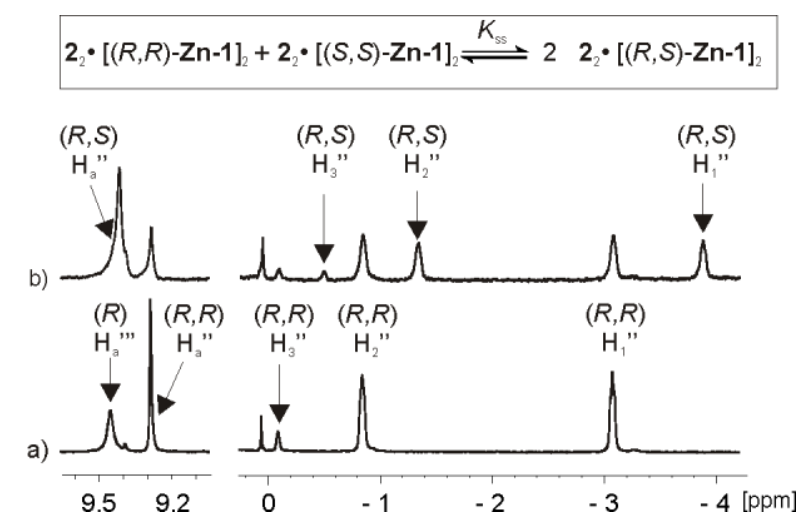

Figure 7 